\title{
Association between comorbid diabetes mellitus and prognosis of patients with sepsis in the intensive care unit: a retrospective cohort study
}

\author{
Shan Lin ${ }^{\#}$, Shanhui Ge", Wanmei He, Mian Zeng \\ Department of Medical Intensive Care Unit, The First Affiliated Hospital of Sun Yat-sen University, Guangzhou, China \\ Contributions: (I) Conception and design: S Lin; (II) Administrative support: S Lin, M Zeng; (III) Provision of study materials or patients: All authors; \\ (IV) Collection and assembly of data: All authors; (V) Data analysis and interpretation: S Lin; (VI) Manuscript writing: All authors; (VII) Final \\ approval of manuscript: All authors. \\ \#These authors contributed equally to this work. \\ Correspondence to: Prof. Mian Zeng. Department of Medical Intensive Care Unit, The First Affiliated Hospital of Sun Yat-sen University/Institute of \\ Pulmonary Diseases, Sun Yat-sen University, No. 58 Zhongshan Road 2, Guangzhou 510080, China. Email: zengmian@mail.sysu.edu.cn.
}

Background: Sepsis patients hospitalized in the intensive care unit (ICU) often have comorbid diabetes mellitus (DM). However, the clinical impact of DM on the clinical outcomes of critically ill sepsis patients has yet to be determined. Therefore, the current study aimed to analyze the association of comorbid DM with the prognosis of sepsis patients in the ICU.

Methods: Data of patients with sepsis and comorbid DM were obtained from a large-scale intensive care database. The primary outcome was 28-day mortality after ICU admission. Associations of comorbid DM with the primary outcome were assessed using a multivariable Cox regression model. Different adjusted models, such as the propensity score method, were used to determine the prognosis of the patients.

Results: Overall, 12,321 sepsis patients were enrolled, including 3,509 (28.48\%) with comorbid DM. After adjusting and matching, we found that comorbid DM was not an independent risk factor for 28day mortality in critically ill sepsis patients and was even associated with lower mortality. Propensity score matching showed a dramatically lower 28-day mortality for sepsis patients with comorbid DM in comparison to patients without comorbid DM [hazard ratio (HR): 0.86, 95\% confidence interval (CI): 0.77-0.97, $\mathrm{P}=0.0167]$. The relationship of comorbid DM with 28-day mortality was broadly consistent for all subgroup variables. In the stratified analysis, a significant interaction was observed only for glucose concentration $(\mathrm{P}<0.0001)$. Patients with comorbid DM and a glucose level of $140-200 \mathrm{mg} / \mathrm{dL}(7.8-11.1 \mathrm{mmol} / \mathrm{L})$ or $\geq 200 \mathrm{mg} / \mathrm{dL}(11.1 \mathrm{mmol} / \mathrm{L})$ had a significantly lower 28 -day mortality rate (HR 0.83, 95\% CI: 0.71-0.98, $\mathrm{P}=0.0250$ and HR: $0.49,95 \%$ CI: $0.38-0.64, \mathrm{P}<0.0001$, respectively).

Conclusions: Critically ill patients with sepsis and comorbid DM were not found to have increased 28-day mortality compared to those without comorbid DM, and may even have a lower risk of mortality. Notably, this association remained in the setting of hyperglycemia.

Keywords: Comorbidity; critical care; diabetes mellitus; prognosis; seps

Submitted Jun 12, 2020. Accepted for publication Sep 30, 2020.

doi: $10.21037 / \mathrm{atm}-20-4360$

View this article at: http://dx.doi.org/10.21037/atm-20-4360

\footnotetext{
$\wedge$ ORCID: 0000-0001-5163-6533.
} 


\section{Introduction}

Diabetes mellitus (DM) is now rapidly rising as a worldwide epidemic. An estimated 451 million people globally are living with the condition, and it is expected to increase to 693 million by 2045 (1). Patients with DM are susceptible to bacterial infections and sepsis (2-4). Moreover, clinical experience has revealed that DM is correlated with fatal infections and poor clinical outcomes, due to factors including immune abnormalities, metabolic disorders, and impaired immune cell function caused by hyperglycemia $(5,6)$. A study from the Netherlands reported that $19.5 \%$ of patients with sepsis in the intensive care unit (ICU) had DM, and these patients had higher rates of hospital and ICU mortality than without DM patients (7). Another retrospective study indicated that $22.7 \%$ of patients with sepsis had DM, although 28-day mortality was comparable between the diabetic and non-diabetic groups (8). Unsurprisingly, DM has emerged as a common comorbidity in patients with sepsis in the ICU.

How comorbid DM affects the clinical endpoints of patients with sepsis in the ICU has not been well established. Additionally, studies linking the outcomes of sepsis patients with critical illness to comorbid DM have proved controversial. Because DM is commonly associated with other comorbidities, this study was aimed to investigate the implications of comorbid DM on the progress of sepsis patients. We present the following article in accordance with the STROBE reporting checklist (available at http:// dx.doi.org/10.21037/atm-20-4360).

\section{Methods}

\section{Patient data}

The study was conducted in accordance with the Declaration of Helsinki (as revised in 2013). This study was carried out using the publicly accessible Medical Information Mart for Intensive Care III database, which contains anonymous data on 58,976 patients hospitalized in Beth Israel Deaconess Medical Center (9). The ethics committees of Beth Israel Deaconess Medical Center and Massachusetts Institute of Technology Affiliates granted access to the database (Record ID: 33460949). Due to the anonymity of the data, patient consent was waived.

From the database, we enrolled patients who were at least 18 years old and diagnosed with sepsis based on a sequential organ failure assessment (SOFA) score of $\geq 2$ points and suspected infection within the first day of ICU admission (10). Patients whose follow-up was $<1$ day and multiple ICU admissions were screened out. The glucose variable for the first of 24 hours after ICU admission was extracted to control confounding by glucose levels. In addition to the exposure factor (comorbid DM or not) and clinical outcomes, the other remaining variables were also extracted, including the Elixhauser Comorbidity Index (SID30) (11), renal replacement therapy on the first day, mechanical ventilation on the first day, infection site, SOFA, age, sex, and other comorbidities. As described previously, structured query language (SQL) was used to extract the data with Navicat (12). The code that facilitates documentation of the website and MIMIC-III is accessible to the public, and input from users is encouraged (13).

\section{Outcomes}

The primary outcome was 28 -day mortality after ICU admission, and the secondary outcome was ICU mortality.

\section{Propensity score models}

In addition to conventional multivariate analysis, we constructed propensity scores for adjustment and matching. In the final model, a covariate was included as potential confounders if it changed the estimate of comorbid DM on 28 -day mortality by $>10 \%$ or was associated markedly with 28-day mortality. The propensity scores were derived using a logistic regression model that included the following baseline covariates: age, sex, SOFA, infection site, renal replacement therapy on the first day, mechanical ventilation on the first day, glucose, depression, drug abuse, alcohol abuse, fluid and electrolyte disorders, solid tumor, obesity, metastatic cancer, lymphoma, acquired immune deficiency syndrome (AIDS), renal failure, liver disease, chronic pulmonary disease, hypertension, peripheral vascular disease, valvular disease, cardiac arrhythmias, congestive heart failure, and other neurological diseases (14). By using a 1:1 matching protocol with the exclusion of replacement (greedy match algorithm), the propensity score matching (PSM) was carried out by a caliper width of 0.01 of the standard deviation of the logit of the propensity score.

\section{Statistical analysis}

Data were expressed as median (interquartile range, IQR) or mean \pm standard deviation for continuous variables, and as numbers and percentages for categorical variables. 
Patient characteristics were analyzed as follows: continuous variables were analyzed using Kruskal-Wallis (or Fisher's exact) tests and categorical variables were to be compared using chi-square tests. Cox regression models were applied to explore the relationship between comorbid DM and 28-day mortality, and logistic regression models were also applied to investigate the association between comorbid DM and ICU mortality. Four different adjusted models were employed to evaluate the clinical outcomes of sepsis patients with comorbid DM. In model I, we adjusted for covariates of age, sex, SOFA, SID30, mechanical ventilation on the first day, infection site, renal replacement therapy on the first day, and glucose. In model II, we used other comorbidities such as depression, drug abuse, alcohol abuse, fluid and electrolyte disorders, obesity, solid tumor, metastatic cancer, lymphoma, neurological disease, hypertension, peripheral vascular disease, valvular disease, cardiac arrhythmias, congestive heart failure, chronic pulmonary disease, liver disease, renal failure, and AIDS instead of SID30, and the other remaining variables were the same as in model I. In models III and IV, we used propensity score adjusted and matching models as described previously. The Kaplan-Meier (KM) method and log-rank tests were utilized to analyze survival differences across sepsis patients with and without comorbid DM. Stratified analyses and interaction tests were performed on the basis of age ( $<65$ and $\geq 65$ years), sex, glucose $(<140,140-200$, and $\geq 200 \mathrm{mg} / \mathrm{dL}$ ), renal replacement therapy on the first day, mechanical ventilation on the first day, infection site and SOFA $(<5,5-10,10-15, \geq 15)$. Both R (http://www. R-project.org, version 3.4.3) and EmpowerStats(R) (www. empowerstats.com, $\mathrm{X} \& \mathrm{Y}$ solutions, Inc., Boston, MA, USA) were used for data analysis. Statistical significance was $\mathrm{P}<0.05$.

\section{Results}

\section{Patient characteristics}

Of the total 12,321 sepsis patients were enrolled, including 3,509 (28.48\%) who had comorbid DM. In the original cohort, the average age was $67.10 \pm 16.42$ years, with no difference between sepsis patients with and without comorbid DM $(67.23 \pm 16.15$ vs. $66.78 \pm 17.06$ years, $\mathrm{P}=0.166$ ). Males accounted for a higher proportion of the patients with DM; however, the difference between the two groups was not significant $(51.78 \%$ vs. $53.06 \%, \mathrm{P}=0.198)$. Patients with comorbid DM showed markedly elevated blood glucose concentrations relative to non-diabetic patients $(165.42 \pm 53.64$ vs. $132.92 \pm 36.64 \mathrm{mg} / \mathrm{dL}, \mathrm{P}<0.001)$. The SOFA, SID30, infection site, and those who needed renal replacement therapy or mechanical ventilation on the first day after ICU admission were similar between the diabetic and non-diabetic groups. For more detailed results are shown in Table 1. After matching, no significant differences were found in the baseline characteristics for the two groups (Table 2).

\section{Clinical outcomes of participants}

In the original cohort (Table 1), hospital mortality (14.88\% vs. $17.19 \%, \mathrm{P}=0.002)$ and ICU mortality $(9.35 \%$ vs. $11.13 \%$, $\mathrm{P}=0.004)$ were lower in diabetic patients than in nondiabetic patients. At the end of the 28 days, 2,263 (18.37\%) of the participants died after ICU admission. Moreover, a lower 28-day mortality rate was observed in patients with DM than in patients without DM (17.30\% vs. 18.79\%); however, the $\mathrm{P}$ value was equal to 0.053 . In the PSM cohort (Table 2), the 28-day mortality, ICU mortality, and inhospital mortality were all considerably lower in patients with comorbid DM than in those without comorbid DM. The length of ICU stay and length of hospital stay were comparable between patients with and without comorbid DM in the original and PSM cohorts. The KM survival curve of the original cohort showed a significant survival benefit in patients with DM within 28 days after ICU admission (log-rank test: $\mathrm{P}=0.044)$ (Figure 1).

\section{Association of comorbid DM with clinical outcomes}

Multivariable Cox regression analysis showed that comorbid DM was not a risk factor for 28-day mortality (HR: 0.91, 95\% CI: 0.83-1.00, $\mathrm{P}=0.0439)$. After adjustments and matching, we found that comorbid DM was still not an independent risk factor and was even associated with lower mortality, with consistent and significant results in the four different models (Table 3). In model IV, the 28-day mortality rate in the comorbid DM group decreased by $14 \%$ compared to the without comorbid DM group (HR: 0.86, 95\% CI: $0.77-0.97, \mathrm{P}=0.0167)$. Similar results were obtained for ICU mortality (Table 3). In model IV, comorbid DM was observed to have a lower risk of ICU mortality (OR: 0.73, 95\% CI: 0.62-0.87, $\mathrm{P}=0.0003$ ).

\section{Interaction and stratified analysis}

The association of comorbid DM with 28-day mortality 
Table 1 Characteristics of the original cohort

\begin{tabular}{|c|c|c|c|c|}
\hline Variables & All patients $(\mathrm{N}=12,321)$ & Without DM $(\mathrm{N}=8,812)$ & With DM $(\mathrm{N}=3,509)$ & $P$ value \\
\hline Sex & & & & 0.198 \\
\hline Male & $6,493(52.70)$ & $4,676(53.06)$ & $1,817(51.78)$ & \\
\hline Female & $5,828(47.30)$ & $4,136(46.94)$ & $1,692(48.22)$ & \\
\hline Glucose (mg/dL) & $142.23 \pm 44.70$ & $132.92 \pm 36.64$ & $165.42 \pm 53.64$ & $<0.001$ \\
\hline Bloodstream & $5,440(44.15)$ & $3,929(44.59)$ & $1,511(43.06)$ & \\
\hline Pulmonary & $807(6.55)$ & $568(6.45)$ & $239(6.81)$ & \\
\hline Abdominal & $265(2.15)$ & $189(2.14)$ & $76(2.17)$ & \\
\hline Urinary tract & $2,596(21.07)$ & $1,867(21.19)$ & $729(20.78)$ & \\
\hline Renal replacement therapy on first day & $604(4.90)$ & $432(4.90)$ & $172(4.90)$ & 0.999 \\
\hline Length of ICU stay (days) & $3.33(1.83-7.86)$ & $3.33(1.83-7.89)$ & $3.33(1.83-7.82)$ & 0.305 \\
\hline Length of hospital stay (days) & $10.85(6.36-19.04)$ & $10.91(6.40-19.39)$ & $10.65(6.29-18.47)$ & 0.128 \\
\hline Elixhauser Comorbidity Index (SID30) & $17.00(8.00-26.00)$ & $17.00(8.00-26.00)$ & $17.00(8.00-26.00)$ & 0.632 \\
\hline 28-day mortality, n (\%) & $2,263(18.37)$ & $1,656(18.79)$ & $607(17.30)$ & 0.053 \\
\hline In-hospital mortality, n (\%) & $2,037(16.53)$ & $1,515(17.19)$ & $522(14.88)$ & 0.002 \\
\hline ICU mortality, n (\%) & $1,309(10.62)$ & $981(11.13)$ & $328(9.35)$ & 0.004 \\
\hline \multicolumn{5}{|l|}{ Other comorbidities, n (\%) } \\
\hline Other neurological disease & $1,719(13.95)$ & $1,308(14.84)$ & $411(11.71)$ & $<0.001$ \\
\hline Chronic pulmonary disease & $2,716(22.04)$ & $1,910(21.67)$ & $806(22.97)$ & 0.118 \\
\hline Liver disease & $1,258(10.21)$ & $901(10.22)$ & $357(10.17)$ & 0.933 \\
\hline Renal failure & $2,226(18.07)$ & $1,160(13.16)$ & $1,066(30.38)$ & $<0.001$ \\
\hline AIDS & $180(1.46)$ & $163(1.85)$ & $17(0.48)$ & $<0.001$ \\
\hline Lymphoma & $309(2.51)$ & $246(2.79)$ & $63(1.80)$ & 0.001 \\
\hline Metastatic cancer & $840(6.82)$ & $677(7.68)$ & $163(4.65)$ & $<0.001$ \\
\hline Solid tumor & $647(5.25)$ & $469(5.32)$ & $178(5.07)$ & 0.575 \\
\hline Obesity & $708(5.75)$ & $310(3.52)$ & $398(11.34)$ & $<0.001$ \\
\hline Fluid and electrolyte disorders & $5,228(42.43)$ & $3,668(41.63)$ & $1,560(44.46)$ & 0.004 \\
\hline Alcohol abuse & $923(7.49)$ & $761(8.64)$ & $162(4.62)$ & $<0.001$ \\
\hline Drug abuse & $405(3.29)$ & $345(3.92)$ & $60(1.71)$ & $<0.001$ \\
\hline Depression & 1,029 (8.35) & $723(8.20)$ & $306(8.72)$ & 0.350 \\
\hline
\end{tabular}

DM, diabetes mellitus; ICU, intensive care unit; SOFA, sequential organ failure assessment; AIDS, acquired immune deficiency syndrome. 
Table 2 Characteristics of the PSM cohort

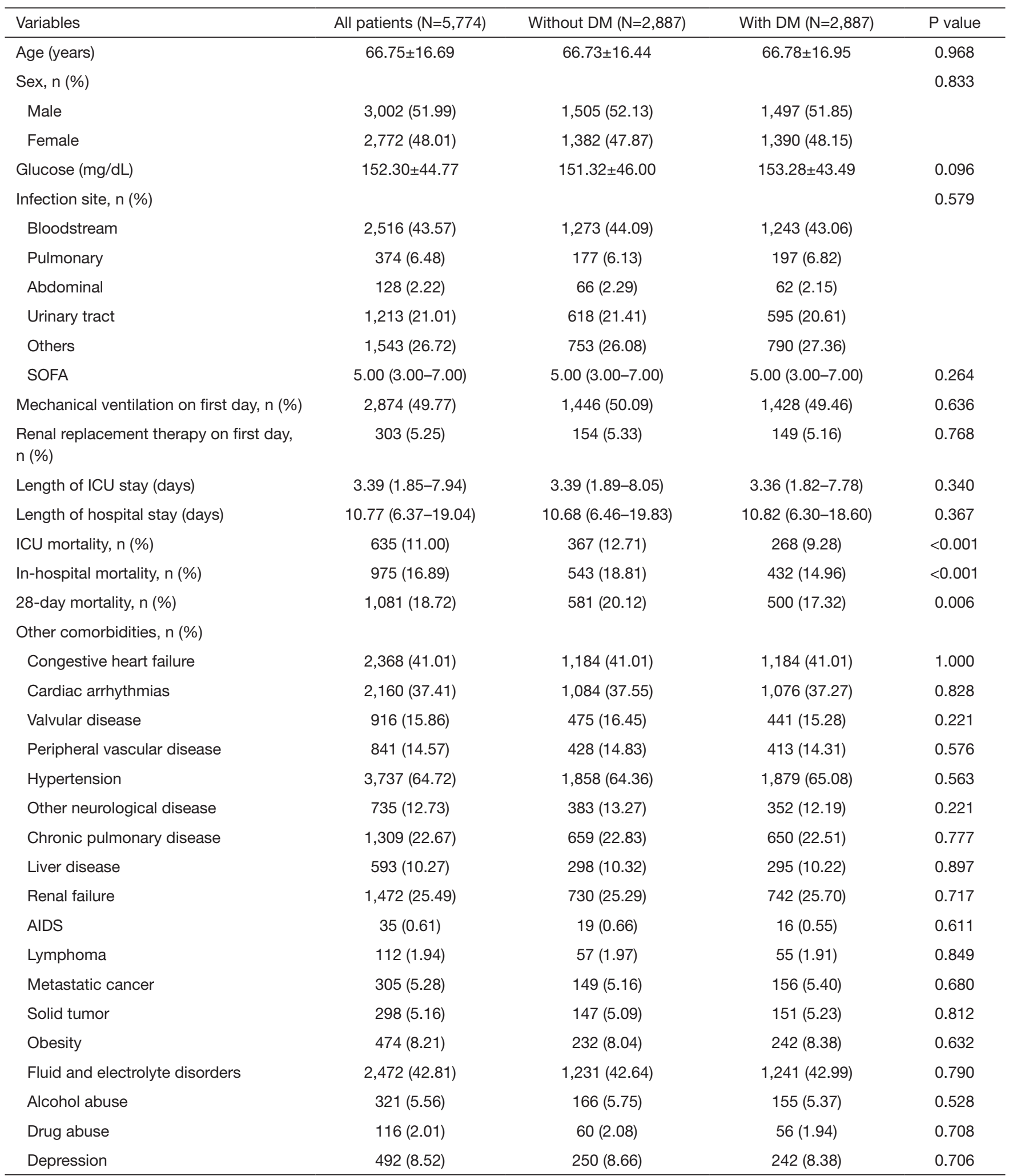

DM, diabetes mellitus; ICU, intensive care unit; SOFA, sequential organ failure assessment; AIDS, acquired immune deficiency syndrome. 

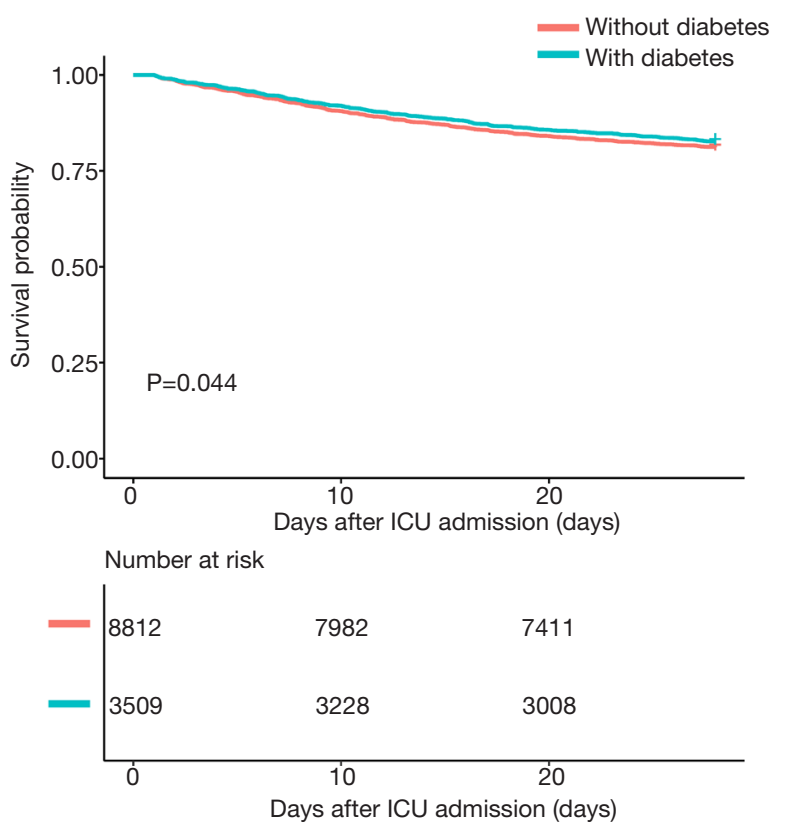

Figure 1 Kaplan-Meier survival curves of the original cohort.

was broadly consistent for all subgroup variables (Table 4 and Figure 2). The correlations between comorbid DM and 28-day mortality was similar for almost strata (Interaction $\mathrm{P}$ value $>0.05)$. A significant interaction was observed only with glucose $(\mathrm{P}<0.0001)$. Patients with comorbid DM and a glucose of $140-200 \mathrm{mg} / \mathrm{dL}(7.8-11.1 \mathrm{mmol} / \mathrm{L})$ or $\geq 200 \mathrm{mg} / \mathrm{dL}(11.1 \mathrm{mmol} / \mathrm{L})$ had a significantly lower risk of 28-day mortality (HR: $0.83,95 \%$ CI: $0.71-0.98, \mathrm{P}=0.0250$ and HR: $0.49,95 \%$ CI: $0.38-0.64, \mathrm{P}<0.0001$, respectively). Additional analyses were conducted of blood glucose concentrations (per $1 \mathrm{mmol} / \mathrm{L}$ ) in patients with and without comorbid DM (Table 5). After adjustments for potential confounders, no dramatic increase was observed in 28-day mortality in the comorbid DM group with increasing blood glucose levels (HR: 1.01, 95\% CI: 0.98-1.03, P=0.6265), whereas there was a remarkable increase in 28-day mortality with increasing blood glucose levels in patients without DM (HR: 1.07, 95\% CI 1.05-1.09, $\mathrm{P}<0.0001$ ).

\section{Discussion}

In this study, we selected a specific study population comprising patients with sepsis and comorbid DM in the ICU from a sizable critical care database, and explored the association between sepsis patients with comorbid DM and clinical outcomes. Comorbid DM was not found to be an independent risk factor for patients with sepsis in the ICU; moreover, a decrease of approximately $14 \%$ was observed in the risk of 28-day mortality in sepsis patients with comorbid DM compared to those without comorbid DM, which remained valid in the setting of hyperglycemia.

While clinical experience points toward an association between DM and poor prognosis, observational studies have yielded conflicting results. However, only a few studies have accurately reported the relationship between prognosis in patients with sepsis and comorbid DM. A study by van Vught et al. (7) identified 41,492 patients with sepsis (including 8,085 with comorbid DM) and indicated that those with comorbid DM had higher 90-day, in-hospital, and crude ICU mortality than those without DM. After adjustment, no relationship was found between DM and the risk of 90-day mortality (7). However, van Vught et al.'s study only evaluated the relationship between 90-day mortality and sepsis patients with comorbid DM, and covariates were not adjusted for in-hospital mortality or ICU mortality. In our study, lower ICU and 28-day mortality were observed for ICU sepsis patients with comorbid diabetes in four different models. Another large sample study of 217,280 patients by de Miguel-Yanes et al. found that inhospital mortality among sepsis patients with comorbid type $2 \mathrm{DM}$ was decreased by $12 \%$ compared to those without type 2 DM (OR: 0.88, 95\% CI: 0.86-0.90) (15). Our results were consistent with those of Miguel-Yanes et al., suggesting that comorbid DM is a potential protective factor in patients with sepsis. van Vught et al. (7) and de MiguelYanes et al. (15) appear to be the only large-sample studies currently available that explore the impact of comorbid DM on the clinical endpoints of patients with sepsis in the ICU. Another study involving 830 patients with sepsis indicated no difference in 28 - or 90 -day mortality between diabetic and non-diabetic patients (8).

With regard to organ dysfunction, there were some studies comparing the prevalence of acute lung injury in sepsis patients with and without comorbid DM, implying a protective role with a lower risk of acute lung injury from comorbid DM $(16,17)$. The findings regarding the relationship between comorbid DM and organ dysfunction were similar between the two studies $(16,17)$; however, we did not analyze their endpoints. It is worth noting that we report for the first time that comorbid DM had a protective effect in patients with sepsis, even with glucose $\geq 200 \mathrm{mg} / \mathrm{dL}$, compared with non-diabetic patients with sepsis. van Vught et al.'s study suggested that admission hyperglycemia is correlated with a high 30-day mortality in all subjects, 
Table 3 Associations between comorbid DM and clinical outcomes

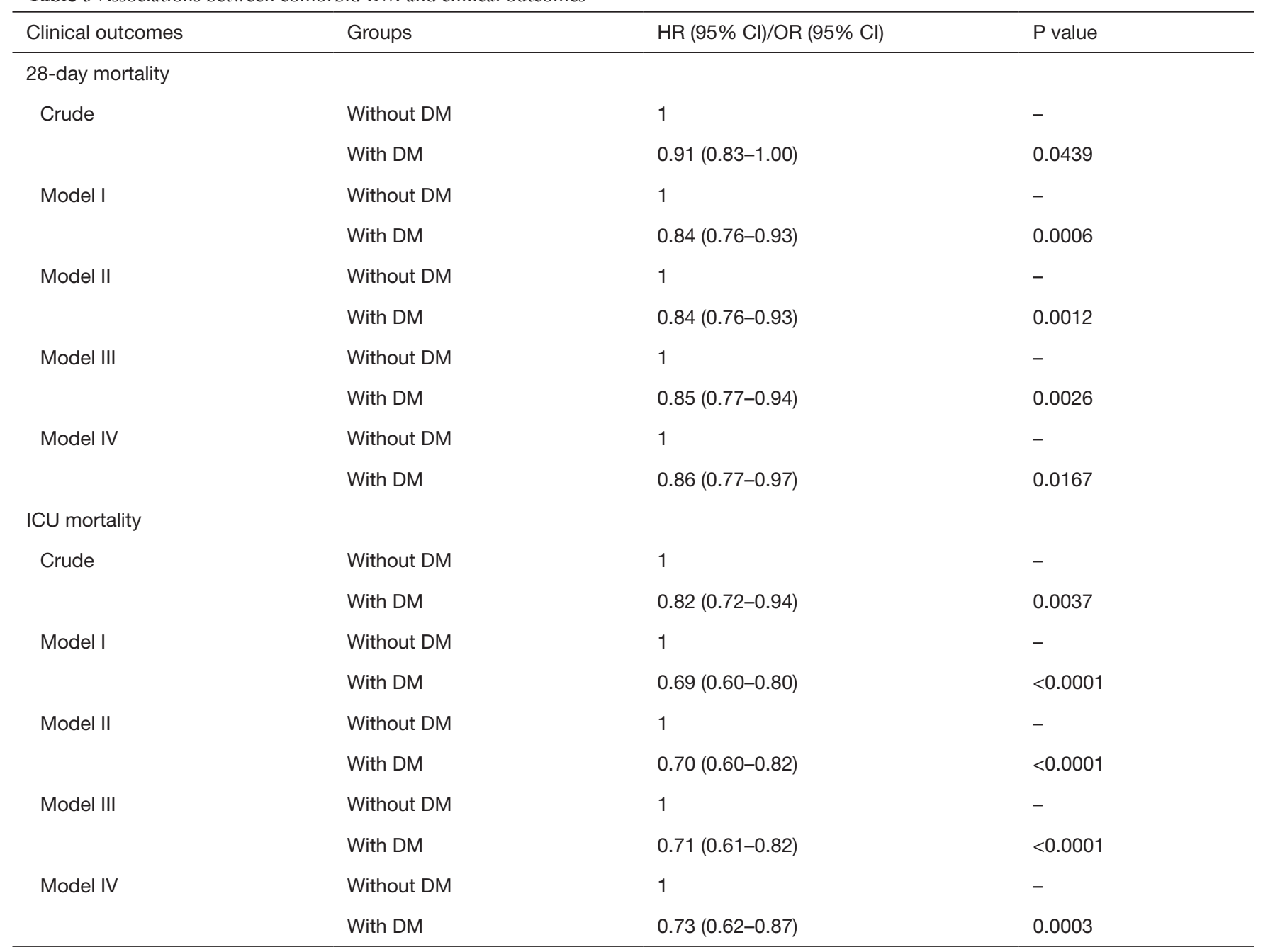

Model I was adjusted by age, sex, SOFA, SID30, infection site, mechanical ventilation on first day, renal replacement therapy on first day, glucose. Model II was adjusted by age, sex, SOFA, infection site, mechanical ventilation on first day, renal replacement therapy on first day, glucose, congestive heart failure, cardiac arrhythmias, valvular disease, peripheral vascular disease, hypertension, other neurological disease, chronic pulmonary disease, liver disease, renal failure, AIDS, lymphoma, metastatic cancer, solid tumor, obesity, fluid and electrolyte disorders, alcohol abuse, drug abuse, and depression. Model III was adjusted by propensity score calculated by age, sex, SOFA, infection site, mechanical ventilation on first day, renal replacement therapy on first day, glucose, congestive heart failure, cardiac arrhythmias, valvular disease, peripheral vascular disease, hypertension, other neurological disease, chronic pulmonary disease, liver disease, renal failure, AIDS, lymphoma, metastatic cancer, solid tumor, obesity, fluid and electrolyte disorders, alcohol abuse, drug abuse, and depression. Model IV was propensity score matching conducted using age, sex, SOFA, infection site, mechanical ventilation on first day, renal replacement therapy on first day, glucose, congestive heart failure, cardiac arrhythmias, valvular disease, peripheral vascular disease, hypertension, other neurological disease, chronic pulmonary disease, liver disease, renal failure, AIDS, lymphoma, metastatic cancer, solid tumor, obesity, fluid and electrolyte disorders, alcohol abuse, drug abuse, and depression. DM, diabetes mellitus; HR, hazard ratio; OR, odds ratio; $\mathrm{Cl}$, confidence interval. 
Table 4 Effect size of comorbid DM on 28-day mortality rate in prespecified and exploratory subgroups in each subgroup

\begin{tabular}{|c|c|c|c|c|c|}
\hline$Y=28$-day mortality & N (\%) & Without DM & With DM (HR, 95\% Cl) & $P$ value & Interaction $\mathrm{P}$ value \\
\hline$<65$ & $5,012(40.68)$ & 1.0 & $1.01(0.83-1.23)$ & 0.9307 & \\
\hline$\geq 65$ & 7,309 (59.32) & 1.0 & $0.79(0.70-0.90)$ & 0.0002 & \\
\hline Sex & & & & & 0.4481 \\
\hline Female & $5,828(47.30)$ & 1.0 & $0.83(0.71-0.97)$ & 0.0190 & \\
\hline Glucose (mg/dL) & & & & & $<0.0001$ \\
\hline$<140$ & $7,037(57.11)$ & 1.0 & $0.99(0.85-1.15)$ & 0.9269 & \\
\hline$\geq 140,<200$ & 3,965 (32.18) & 1.0 & $0.83(0.71-0.98)$ & 0.0250 & \\
\hline No & $6,189(50.23)$ & 1.0 & $0.80(0.69-0.93)$ & 0.0031 & \\
\hline Yes & $6,132(49.77)$ & 1.0 & $0.89(0.76-1.03)$ & 0.1048 & \\
\hline Renal replacement therapy on first day & & & & & 0.8129 \\
\hline No & $11,717(95.10)$ & 1.0 & $0.83(0.75-0.93)$ & 0.0010 & \\
\hline Yes & $604(4.90)$ & 1.0 & $0.98(0.65-1.48)$ & 0.9169 & \\
\hline Infection site & & & & & 0.8896 \\
\hline Bloodstream & $5,440(44.15)$ & 1.0 & $0.81(0.69-0.94)$ & 0.0065 & \\
\hline Pulmonary & $807(6.55)$ & 1.0 & $0.77(0.51-1.16)$ & 0.2084 & \\
\hline$\geq 5,<10$ & $5,280(42.85)$ & 1.0 & $0.82(0.70-0.95)$ & 0.0110 & \\
\hline$\geq 10,<15$ & $1,197(9.72)$ & 1.0 & $1.08(0.85-1.37)$ & 0.5287 & \\
\hline$\geq 15$ & $178(1.44)$ & 1.0 & $0.46(0.22-0.98)$ & 0.0451 & \\
\hline
\end{tabular}

Adjusted by age, sex, SOFA, infection site, mechanical ventilation on first day, renal replacement therapy on first day, glucose, congestive heart failure, cardiac arrhythmias, valvular disease, peripheral vascular disease, hypertension, other neurological disease, chronic pulmonary disease, liver disease, renal failure, AIDS, lymphoma, metastatic cancer, solid tumor, obesity, fluid and electrolyte disorders, alcohol abuse, drug abuse, and depression except for the subgroup variable. DM, diabetes mellitus; HR, hazard ratio; CI, confidence interval. 


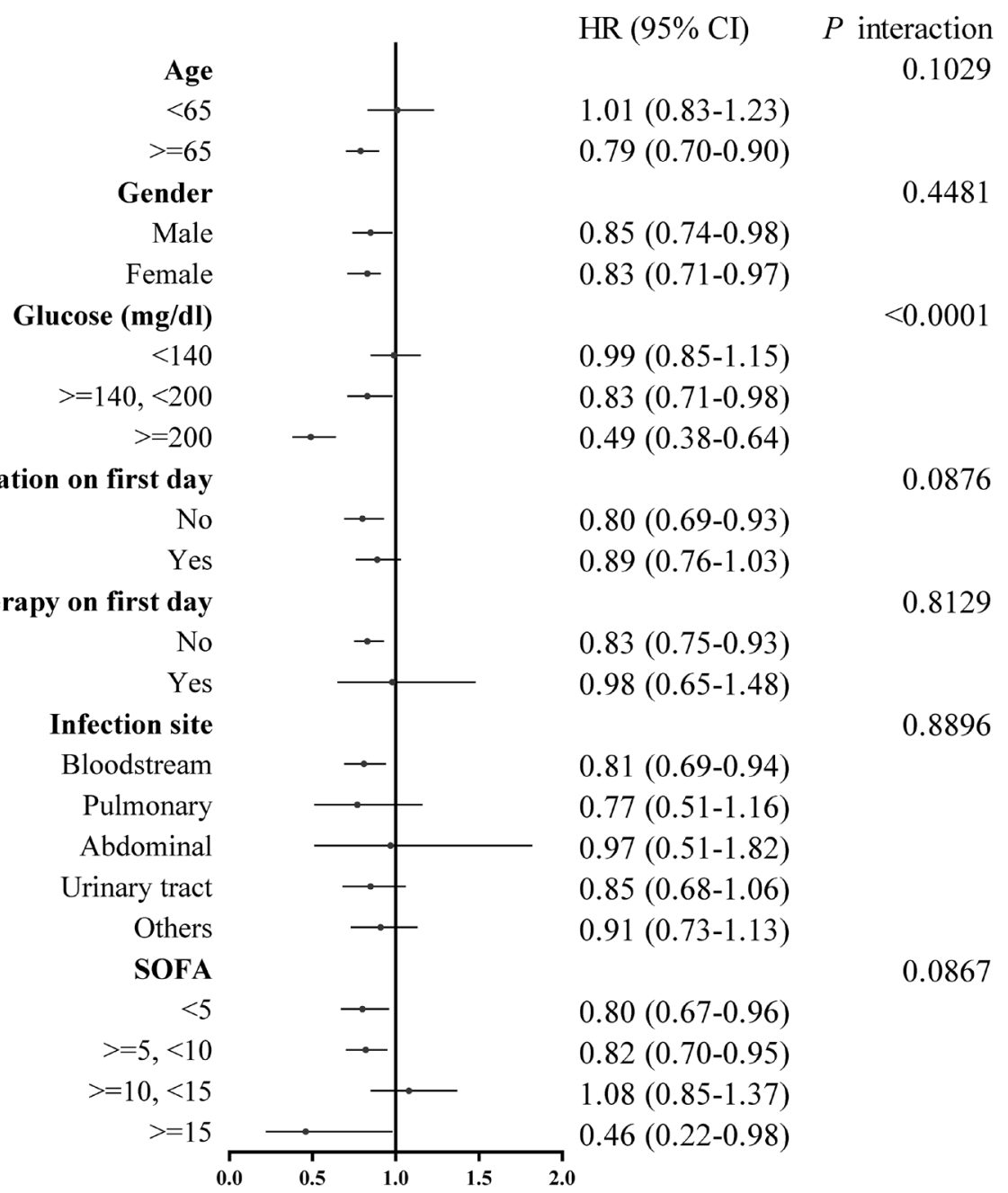

Figure 2 Forest plot of comorbid DM on 28-day mortality in prespecified and exploratory subgroups in each subgroup. DM, diabetes mellitus; ICU, intensive care unit; SOFA, sequential organ failure assessment; HR, hazard ratio; CI, confidence interval.

Table 5 Effect of blood glucose on 28-day mortality in patients with and without comorbid DM

\begin{tabular}{lccc}
\hline Clinical outcomes (28-day mortality) & Groups & HR (95\% Cl) & P value \\
\hline Crude (per 1 mmol/L) & Without DM & $1.08(1.05-1.10)$ & $<0.0001$ \\
& With DM & $1.00(0.97-1.03)$ & 0.9565 \\
Adjusted model (per 1 mmol/L) & Without DM & $1.07(1.05-1.09)$ & $<0.0001$ \\
& With DM & $1.01(0.98-1.03)$ & 0.6265
\end{tabular}

Adjusted for age, sex, SOFA, infection site, mechanical ventilation on first day, renal replacement therapy on first day, congestive heart failure, cardiac arrhythmias, valvular disease, peripheral vascular disease, hypertension, other neurological disease, chronic pulmonary disease, liver disease, renal failure, AIDS, lymphoma, metastatic cancer, solid tumor, obesity, fluid and electrolyte disorders, alcohol abuse, drug abuse, and depression. DM, diabetes mellitus; HR, hazard ratio; $\mathrm{Cl}$, confidence interval. 
although that study had a small sample size and involved only 218 sepsis patients with comorbid DM (18). van Niekerk et al. believed that glucose is essential for energy production and plays a central role in maintaining the function of activated immune cells (19), which may explain the protective effect of hyperglycemia in sepsis patients with comorbid DM.

All of these studies, including ours, reveal a complex picture of the interaction between DM and the prognosis of sepsis. Diabetes causes immune dysfunction as well as metabolic disorders, including hyperglycemia. Furthermore, DM treatments (e.g., insulin, metformin, sulfonylureas, and thiazolidinediones), together with the effect of DM on the immune system, may modify the host response to sepsis and clinical outcomes $(20,21)$. More research is urgently needed to completely understand the factors influencing the overall effect of DM on the prognosis of sepsis.

There were also limitations to our study. No data were available regarding the type of DM (e.g., type 1 or type $2 \mathrm{DM}$ ) or medications (e.g., insulin, metformin, etc.); consequently, we could not assess their effects on the patients in our study. Furthermore, the duration and severity of DM, as well as medications prescribed prior to hospitalization, were also not available from the data, so the effects of these factors could not be measured either. Nevertheless, a strength of this study is that multiple models were applied to adjust for potential confounders (22). Other limitations include the selection bias resulting from the study's retrospective nature, single-center design, and low external validity.

\section{Conclusions}

In this study, comorbid DM was not an independent risk factor for 28-day mortality or ICU mortality in patients with sepsis, and may instead be related to a lower mortality rate. Notably, the mortality-reducing effect persisted at glucose concentrations of $140-200 \mathrm{mg} / \mathrm{dL}$ as well as $\geq 200 \mathrm{mg} / \mathrm{dL}$, indicating that a personalized glycemic control strategy in critically ill sepsis patients, both diabetic and non-diabetic, as a potential protective mechanism warrants further investigation.

\section{Acknowledgments}

In addition, we would like to thank AME Editing Service (editing.amegroups.com) for English language editing.
Funding: This work was supported by the National Natural Science Foundation of China (grant no. 81670066), the Major Science and Technology Planning Project of Guangdong Province, China (grant no. 2016A020216009), the Critical Care Research Funding of the Aesculap Academy (2017), and the Guangdong Basic and Applied Basic Research Foundation, China (grant no. 2019A1515011198).

\section{Footnote}

Reporting Checklist: The authors have completed the STROBE reporting checklist. Available at http://dx.doi. org/10.21037/atm-20-4360

Data Sharing Statement: The raw data are from a third-party dataset available from MIMIC-III, a freely accessible critical care database. Reproduction of this data is not permitted according to the Data Use Agreement of the database but access can be requested here: https://mimic.physionet.org/ gettingstarted/access.

Conflicts of Interest: All authors have completed the ICMJE uniform disclosure form (available at http://dx.doi. org/10.21037/atm-20-4360). The authors have no conflicts of interest to declare.

Ethical Statement: The authors are accountable for all aspects of the work in ensuring that questions related to the accuracy or integrity of any part of the work are appropriately investigated and resolved. The study was conducted in accordance with the Declaration of Helsinki (as revised in 2013). The access to the database has been approved by the institutional review boards of Beth Israel Deaconess Medical Center and Massachusetts Institute of Technology Affiliates (Record ID: 33460949).

Open Access Statement: This is an Open Access article distributed in accordance with the Creative Commons Attribution-NonCommercial-NoDerivs 4.0 International License (CC BY-NC-ND 4.0), which permits the noncommercial replication and distribution of the article with the strict proviso that no changes or edits are made and the original work is properly cited (including links to both the formal publication through the relevant DOI and the license). See: https://creativecommons.org/licenses/by-nc$\mathrm{nd} / 4.0 \%$. 


\section{References}

1. Cho NH, Shaw JE, Karuranga S, et al. IDF Diabetes Atlas: Global estimates of diabetes prevalence for 2017 and projections for 2045. Diabetes Res Clin Pract 2018;138:271-81.

2. Shah BR, Hux JE. Quantifying the risk of infectious diseases for people with diabetes. Diabetes Care 2003;26:510-3.

3. Muller LM, Gorter KJ, Hak E, et al. Increased risk of common infections in patients with type 1 and type 2 diabetes mellitus. Clin Infect Dis 2005;41:281-8.

4. Benfield T, Jensen J S, Nordestgaard B G. Influence of diabetes and hyperglycaemia on infectious disease hospitalisation and outcome. Diabetologia 2007;50:549-54.

5. Schuetz P, Castro P, Shapiro NI. Diabetes and sepsis: preclinical findings and clinical relevance. Diabetes care 2011;34:771-8.

6. Frydrych LM, Fattahi F, He K, et al. Diabetes and sepsis: risk, recurrence, and ruination. Front Endocrinol (Lausanne) 2017;8:271.

7. van Vught L A, Holman R, de Jonge E, et al. Diabetes is not associated with increased 90-day mortality risk in critically ill patients with sepsis. Crit Care Med 2017;45:e1026-35.

8. Stegenga ME, Vincent JL, Vail GM, et al. diabetes does not alter mortality or hemostatic and inflammatory responses in patients with severe sepsis. Crit Care Med 2010;38:539-45.

9. Johnson AE, Pollard TJ, Shen L, et al. MIMIC-III, a freely accessible critical care database. Sci Data 2016;3:160035.

10. Singer M, Deutschman CS, Seymour CW, et al. The Third International Consensus Definitions for Sepsis and Septic Shock (Sepsis-3). JAMA 2016;315:801-10.

11. Steiner C, Elixhauser A, Schnaier J. The healthcare cost and utilization project: an overview. Eff Clin Pract 2002;5:143-51.

12. Lin $\mathrm{S}, \mathrm{Ge} \mathrm{S}, \mathrm{He} \mathrm{W}$, et al. Association of delayed time in

Cite this article as: Lin S, Ge S, He W, Zeng M. Association between comorbid diabetes mellitus and prognosis of patients with sepsis in the intensive care unit: a retrospective cohort study. Ann Transl Med 2021;9(1):22. doi: 10.21037/atm-20-4360 the emergency department with the clinical outcomes for critically ill patients. QJM 2020. [Epub ahead of print].

13. Johnson AE, Stone DJ, Celi LA, Pollard TJ. The MIMIC code repository: enabling reproducibility in critical care research. J Am Med Inform Assoc 2018;25:32-9.

14. Rubin DB. Using propensity scores to help design observational studies: application to the tobacco litigation. Health Serv Outcomes Res Methodol 2001;2:169-88.

15. de Miguel-Yanes J M, Méndez-Bailón M, Jiménez-García $\mathrm{R}$, et al. Trends in sepsis incidence and outcomes among people with or without type 2 diabetes mellitus in Spain (2008-2012). Diabetes Res Clin Pract 2015;110:266-75.

16. Esper AM, Moss M, Martin GS. The effect of diabetes mellitus on organ dysfunction with sepsis: an epidemiological study. Crit Care 2009;13:R18.

17. Moss M, Guidot DM, Steinberg KP, et al. Diabetic patients have a decreased incidence of acute respiratory distress syndrome. Crit Care Med 2000;28:2187-92.

18. van Vught LA, Wiewel MA, Klein Klouwenberg P, et al. Admission hyperglycemia in critically ill sepsis patients: association with outcome and host response. Crit Care Med 2016;44:1338-46.

19. van Niekerk G, Davis T, Engelbrecht AM. Hyperglycaemia in critically ill patients: the immune system's sweet tooth. Crit Care 2017;21:202.

20. Koh GC, Peacock SJ, Van Der Poll T, et al. The impact of diabetes on the pathogenesis of sepsis. Eur J Clin Microbiol Infect Dis 2012;31:379-88.

21. van Vught LA, Scicluna BP, Hoogendijk AJ, et al. Association of diabetes and diabetes treatment with the host response in critically ill sepsis patients. Crit Care 2016;20:252.

22. Kurth T, Walker AM, Glynn RJ, et al. Results of multivariable logistic regression, propensity matching, propensity adjustment, and propensity-based weighting under conditions of nonuniform effect. Am J Epidemiol 2006;163:262-70. 